УДК 517.957

\title{
On the Properties of Solutions of Multidimensional Nonlinear Filtration Problem with Variable Density and Nonlocal Boundary Condition in the Case of Fast Diffusion
}

\author{
Zafar R. Rakhmonov* \\ National University of Uzbekistan \\ 100174, University street, 4. Tashkent
}

Uzbekistan

Received 30.08.2015, received in revised form 04.12.2015, accepted 12.01.2016

The conditions of global existence of solutions of a nonlinear filtration problem in an inhomogeneous medium are investigated in this paper. Various techniques such as the method of standard equations, selfsimilar analysis and the comparison principle are used to obtain results. The influence of inhomogeneous medium on the evolution process is analyzed. The critical global existence exponent and the critical Fujita exponent are obtained. Asymptotic behavior of solutions in the case of the global solvability is established.

Keywords: filtration, asymptotics, critical exponent, inhomogeneous density.

DOI: $10.17516 / 1997-1397-2016-9-2-225-234$.

We consider the qualitative properties of solutions of the following quasilinear parabolic equation

$$
\rho(x) u_{t}=\nabla\left(\left|\nabla u^{m}\right|^{p-2} \nabla u^{m}\right), \quad(x, t) \in R_{+}^{N} \times(0,+\infty),
$$

with nonlinear boundary condition

$$
-\left|\nabla u^{m}\right|^{p-2} \frac{\partial u^{m}}{\partial x_{1}}(0, t)=u^{q}, \quad x_{1}=0, t>0,
$$

and initial condition

$$
u(x, 0)=u_{0}(x) \geqslant 0, \quad x \in R_{+}^{N},
$$

where $R_{+}^{N}=\left\{\left(x_{1}, x^{\prime}\right) \mid x^{\prime} \in R^{N-1}, x_{1}>0\right\}, \rho(x)=(1+|x|)^{n}, n>-p, m>1, q>0,1<p<$ $1+1 / m$ and $u_{0}(x)$ is a nontrivial, nonnegative, bounded and sufficiently smooth function.

Problem (1)-(3) appears in various applications [2]. Such problem describes many physical, chemical, biological and other processes. For example, equation (1) arises in mathematical modelling of reaction-diffusion process in nonlinear media, fluid flows through porous media, dynamics of biological populations, polytropic filtration, synergy structures and various other phenomena $[2,4]$.

Equation (1) is a parabolic equation with inhomogeneous density [2]. In the case $1<p<$ $1+1 / m$ it corresponds to the fast diffusion equation [2]. Problem (1)-(3) has been intensively studied by many authors (see $[3,5-14]$ and references therein) for various values of numerical parameters. Galaktionov and Levine [13] considered problem (1)-(3) in the case $m=1, n=0$, $N=1$. They have proved that if $2(p-1) / p<q<2(p-1)$ then all solutions of problem (1) $-(3)$ become unbounded in finite time. They also found that solutions of problem (1)-(3) have the following properties:

- if $0<q \leqslant 2(p-1) / p$ then global solution of problem (1)-(3) exists;

- if $q>2(p-1)$ then problem (1)-(3) admits nontrivial global solutions with small initial data.

*zraxmonov@inbox.ru

(C) Siberian Federal University. All rights reserved 
Here $q_{0}=2(p-1) / p$ is the critical global existence exponent and $q_{c}=2(p-1)$ is the critical Fujita exponent [3]. Similar results were established for various nonlinear parabolic equations [3, $4,8,14,15]$.

Some properties of solutions of problem (1)-(3) at $\rho(x)=1, N=1, m=1$ were studied by Zejia, Jingxue and Chunpeng [5]. In the case of slow diffusion it has been proved that solution of problem (1)-(3) is global in time when $0<q \leqslant(m+1)(p-1) / p$. Moreover, they also proved that $q_{c}=(m+1)(p-1)$ is the critical Fujita exponent. If $(m+1)(p-1) / p<q<q_{c}$ then all solutions become unbounded in finite time but if $q>q_{c}$ global solutions exist. In the case of fast diffusion similar results have been obtained [6,7].

Problem (1)-(3) has been studied in the case of slow diffusion $(p>2)$ at $m=1, n=0$ [14]. The critical global existence exponent $q_{0}=2(p-1) / p$ and the critical Fujita exponent $q_{c}=$ $(1+1 / N)(p-1)$ were obtained with the use of upper bound and lower bound solutions.

In this paper, we investigate the conditions of global solvability and non-solvability of solutions of problem (1)-(3) on the basis of self-similar analysis and the method of standard equations [1]. We also study the influence of inhomogeneity of the medium on the process under consideration. We construct various self-similar solutions of problem (1)-(3) in the case of global solvability which are the asymptotics of solutions of problem (1)-(3). The methods of choosing an appropriate initial approximation for the iterative process are presented. They conserve qualitative properties of problem (1)-(3). In contrast to [5-7, 9, 11-15], estimates of the solutions, the critical Fujita exponent and critical global existence exponent are obtained for multidimensional problem (1)-(3) in the case of inhomogeneous medium. Problem (1)-(3) is considered more fully in this paper. The asymptotics of solutions for the sufficiently large values of the argument are obtained. Numerical calculations show the fast convergence to the exact solution.

The main results of the paper are the following theorems.

Let us introduce the following designations

$$
q_{0}=\frac{(m(n+1)+1)(p-1)}{p+n}, \quad q_{c}=m(p-1)+\frac{p-1}{N+n} .
$$

Theorem 1. If $0 \leqslant q \leqslant q_{0}$ then each solution of problem (1)-(3) is global solution.

Proof. Let

$$
u_{+}(x, t)=e^{L t} g(\xi), \quad g(\xi)=M\left(K+e^{-\xi}\right)^{1 / m}, \quad \xi=\left(1+x_{1}\right) e^{J t}, \quad x_{i}=0, \quad i=2,3, \ldots, N,
$$

where

$$
\begin{aligned}
& L=\frac{(p-1)(p+n) m M^{m(p-1)-1} K^{-\frac{1+m}{m}}}{m K(p+n)-(1-m(p-1)) e^{-1}}, \quad J=\frac{1-m(p-1)}{p+n} L, \\
& M=e^{\frac{p-1}{m(m(p-1)-q)}}\left(K+e^{-1}\right)^{\frac{q}{m(m(p-1)-q)}}, \quad M^{m}\left(K+e^{-1}\right)>\left\|u_{0}\right\|_{\infty}^{m} .
\end{aligned}
$$

It is easy to obtain that

$$
\begin{gathered}
-\left.\left|\frac{\partial u_{+}^{m}}{\partial x}\right|^{p-2} \frac{\partial u_{+}^{m}}{\partial x}\right|_{x_{1}=0}=-e^{(p-1)(L m+J) t}\left|\left(g^{m}\right)^{\prime}\right|^{p-2}\left(g^{m}\right)^{\prime}(1)=M^{m(p-1)} e^{(L m+J)(p-1) t} e^{-(p-1)} \\
\frac{\partial}{\partial x}\left(\left|\frac{\partial u_{+}^{m}}{\partial x}\right|^{p-2} \frac{\partial u_{+}^{m}}{\partial x}\right)(x, t)=e^{(L m(p-1)+J p) t}\left|\left(g^{m}\right)^{\prime}\right|^{p-2}\left(g^{m}\right)^{\prime}(\xi)= \\
=(p-1) M^{m(p-1)} e^{(L m(p-1)+J p) t} e^{-(p-1) \xi}
\end{gathered}
$$




$$
\begin{aligned}
& \rho(x) \frac{\partial u_{+}}{\partial t}(x, t)=e^{(L-J n) t} \xi^{n}\left(L g(\xi)+J \xi g^{\prime}(\xi)\right)= \\
= & e^{(L-J n) t} M \xi^{n}\left(L\left(K+e^{-\xi}\right)^{\frac{1}{m}}-\frac{J}{m} \xi e^{-\xi}\left(K+e^{-\xi}\right)^{\frac{1}{m}-1}\right) \geqslant e^{(L-J n) t} M K^{\frac{1}{m}}\left(L-\frac{J e^{-1}}{m K}\right) .
\end{aligned}
$$

Let us show that function $u_{+}(x, t)$ is upper bound solution of problem (1)-(3). According to the comparison principle, the function should satisfy the following inequality:

$$
\begin{aligned}
\rho(x) \frac{\partial u_{+}}{\partial t} & \geqslant \frac{\partial}{\partial x}\left(\left|\frac{\partial u_{+}^{m}}{\partial x}\right|^{p-2} \frac{\partial u_{+}^{m}}{\partial x}\right),(x, t) \in R_{+}^{N} \times(0,+\infty), \\
& -\left|\frac{\partial u_{+}^{m}}{\partial x}\right|^{p-2} \frac{\partial u_{+}^{m}}{\partial x}(0, t) \geqslant u_{+}^{q}(0, t), t>0 .
\end{aligned}
$$

Taking into account the definition of $M, L, K$ and $J$, it is not difficult to verify that if $0 \leqslant q \leqslant q_{0}$ then inequalities (4) and (5) are true. Hence, we have $u_{+}(x, 0) \geqslant u_{0}(x)$ and $u_{+}(0,0)>u_{0}(0)$. Thus, according to the comparison principle, Theorem 1 is proved.

Theorem 2. If $q>q_{c}$ then problem (1)-(3) has nontrivial global solutions with small initial data.

Proof. Let us construct a global self-similar upper bound solution of problem (1)-(3) in the form

$$
u_{+}(t, x)=(T+t)^{-\gamma} f(\xi),
$$

in the domain $\mathbb{Q}=\left\{(x, t): x \in R_{+}^{N}, 0<t<+\infty\right\}$, where $\xi=|\zeta|, \zeta_{i}=\left(1+x_{i}\right)(T+t)^{-\sigma}$, $i=1,2, \ldots, N, \gamma=\frac{p-1}{q(p+n)-(p-1)(m(n+1)+1)}, \sigma=\frac{q-m(p-1)}{q(p+n)-(p-1)(m(n+1)+1)}$.

For function $u_{+}(x, t)$ to be upper bound solution of problem (1)-(3), by the comparison principle, the function $f(\xi)$ should satisfy the following inequalities $[3,6,7]$

$$
\begin{gathered}
\xi^{1-N} \frac{d}{d \xi}\left(\xi^{N-1}\left|\frac{d f^{m}}{d \xi}\right|^{p-2} \frac{d f^{m}}{d \xi}\right)+\sigma \xi^{n+1} \frac{d f}{d \xi}+\gamma \xi^{n} f \leqslant 0 \\
-\left|\left(f^{m}\right)^{\prime}\right|^{p-2}\left(f^{m}\right)^{\prime}(1) \geqslant f^{q}(1) .
\end{gathered}
$$

Let us set

$$
f(\xi)=\left(a+b \xi^{\frac{p+n}{p-1}}\right)^{-\frac{p-1}{1-m(p-1)}},
$$

where $b=\frac{1-m(p-1)}{m(p+n)} \sigma^{1 /(p-1)}, a>0$. Using (9), inequality (7) is transformed into

$$
-\left(\frac{(q-m(p-1))(N+n)}{q(p+n)-(p-1)(m(n+1)+1)}-\frac{p-1}{q(p+n)-(p-1)(m(n+1)+1)}\right) \xi^{n} f \leqslant 0 .
$$

It is easy to check that under the conditions of Theorem 2 inequality (10) is always true. Now we consider condition (8). After substituting function (9) into (8), we obtain the following expression:

$$
\sigma(a+b)^{-\frac{p-1}{1-m(p-1)}} \geqslant(a+b)^{-\frac{q(p-1)}{1-m(p-1)}} .
$$

It is valid when

$$
a+b \leqslant \sigma^{-\frac{1-m(p-1)}{(q-1)(p-1)}} .
$$


In conclusion, we note that self-similar solution (6), (9) is the upper bound solution of problem $(1)-(3)$ and $u(x, t) \leqslant u_{+}(x, t)$ in $\mathbb{Q}$. By the comparison principle, the solution of problem $(1)-(3)$ is global solution if the initial data $u_{0}(x)$ is small enough.

Theorem 3. If $q>q_{0}$ then solution of problem (1)-(3) with appropriately large initial data becomes unbounded in finite time.

Proof. To prove the nonexistence of global solutions, we represent a self-similar lower bound solution of problem (1)-(3) in the form

$$
u_{-}(x, t)=(T-t)^{-\gamma} \vartheta(\xi)
$$

where $\xi=|\zeta|, \zeta_{i}=\left(1+x_{i}\right)(T-t)^{-\sigma}, i=1,2, \ldots, N$ and function $\vartheta(\xi)$ satisfies the equation

$$
\xi^{1-N} \frac{d}{d \xi}\left(\xi^{N-1}\left|\frac{d \vartheta^{m}}{d \xi}\right|^{p-2} \frac{d \vartheta^{m}}{d \xi}\right)-\sigma \xi^{n+1} \frac{d \vartheta}{d \xi}-\gamma \xi^{n} \vartheta=0
$$

$\gamma$ and $\sigma$ are given above.

Let us show that function $u_{-}(x, t)$ defined by (12) is a lower bound solution. Then, according to the comparison principle, function $\vartheta(\xi)$ should satisfy the following inequalities

$$
\begin{gathered}
\xi^{1-N} \frac{d}{d \xi}\left(\xi^{N-1}\left|\frac{d \vartheta^{m}}{d \xi}\right|^{p-2} \frac{d \vartheta^{m}}{d \xi}\right)-\sigma \xi^{n+1} \frac{d \vartheta}{d \xi}-\gamma \xi^{n} \vartheta \geqslant 0 \\
-\left|\left(\vartheta^{m}\right)^{\prime}\right|^{p-2}\left(\vartheta^{m}\right)^{\prime}(1) \leqslant \vartheta^{q}(1)
\end{gathered}
$$

Let us set

$$
\vartheta(\xi)=A\left(a+\xi^{\frac{p+n}{p}}\right)^{-\frac{p-1}{1-m(p-1)}}
$$

where $a$ and $A$ are positive constants to be determined. Upon substituting $\vartheta(\xi)$ into (14) and (15), we obtain the following inequalities

$$
\begin{aligned}
& j_{1}\left(\xi^{\frac{p+n}{p}}\right)^{2}+j_{2} \xi^{\frac{p+n}{p}}-j_{3} \geqslant 0 \\
& A^{m(p-1)}\left(\frac{m(p-1)(p+n)}{p(1-m(p-1))}\right)^{p-1}(a+1)^{\frac{p-1}{1-m(p-1)}} \leqslant A^{q}(a+1)^{-\frac{q(p-1)}{1-m(p-1)}},
\end{aligned}
$$

where

$$
\begin{aligned}
& j_{1}=\frac{(p+n)(p-1)}{p(1-m(p-1))} \sigma-\gamma, j_{2}=\left(\frac{(p+n)(p-1)}{p(1-m(p-1))}-\lambda\right)\left(\frac{m(p+n)(p-1)}{p(1-m(p-1))}\right)^{p-1} A^{m(p-1)-1}-\gamma a \\
& j_{3}=a \lambda\left(\frac{m(p+n)(p-1)}{p(1-m(p-1))}\right)^{p-1} A^{m(p-1)-1}, \lambda=N-1+\frac{n(p-1)}{p} .
\end{aligned}
$$

Now we consider conditions for constants $A$ and $a$. Note that inequality (16) is satisfied only under the following conditions:

a) if $j_{1}>0$ then (16) implies that $q>q_{0}$;

b) if $j_{2} \geqslant 0$ then (16) implies $\left(\frac{(p+n)(p-1)}{p(1-m(p-1))}-\lambda\right)\left(\frac{m(p+n)(p-1)}{p(1-m(p-1))}\right)^{p-1} A^{m(p-1)-1} \geqslant \gamma a$; c) if (16) holds at $\xi \rightarrow 1_{+}$then we have $j_{1}+j_{2}+j_{3} \geqslant 0$. 
It follows from (17) that

$$
A^{q-m(p-1)}(a+1)^{\frac{(q-1)(p-1)}{m(p-1)-1}} \geqslant\left(\frac{m(p-1)(p+n)}{p(1-m(p-1))}\right)^{p-1}
$$

Thus, there are constants $A$ and $a$ that satisfy inequalities a)-c) and (18). The comparison principle implies that $u(x, t) \leqslant u(x, t)$ in $R_{+}^{N} \times(0, T)$. Therefore, solution $u(x, t)$ of problem (1)-(3) with appropriately large initial data becomes unbounded in finite time.

Theorem 4. If $q_{0}<q<q_{c}$ then any nontrivial solution of problem (1)-(3) becomes unbounded in finite time.

Proof. The proof of Theorem 4 is based on the function

$$
u_{1}(x, t)=(\tau+t)^{-\frac{N+n}{N m(p-1)+p-N}} H(\xi), \xi=|\zeta|, \zeta=\left(1+x_{i}\right)(\tau+t)^{-\frac{1}{N m(p-1)+p-N}}
$$

where $\tau \geqslant 0$,

$$
H(\varsigma)=\left(c_{1}+b_{1} \xi^{\frac{p+n}{p-1}}\right)_{+}^{-\frac{p-1}{1-m(p-1)}}, \quad b_{1}=\frac{1-m(p-1)}{(p+n) m}\left(\frac{1}{m(p-1)(n+1)+p-1}\right)^{\frac{1}{p-1}} .
$$

It is easy to verify that $\xi \in\{\xi>0 \mid H(\xi) \geqslant 0\}$ satisfies the following equation

$$
\xi^{1-N} \frac{d}{d \xi}\left(\xi^{N-1}\left|\frac{d H^{m}}{d \xi}\right|^{p-2} \frac{d H^{m}}{d \xi}\right)+\frac{1}{S} \xi^{n+1} \frac{d H}{d \xi}+\frac{n+1}{S} \xi^{n} H=0,
$$

where $S=m(p-1)(n+1)+p-1$.

Then we choose $c_{1}>0$ and $t_{0} \geqslant 0$ such that

$$
u\left(x, t_{0}\right) \geqslant u_{1}\left(x, t_{0}\right), x \in R_{+}^{N} .
$$

Hence, it follows from the comparison principle that

$$
u(x, t) \geqslant u_{1}(x, t),(x, t) \in R_{+}^{N} \times\left(t_{0},+\infty\right) .
$$

We show that there exists $t_{*}>t_{0}$ and a sufficiently large $T$ that

$$
u_{1}\left(x, t_{*}\right) \geqslant u_{-}(x, 0), x \in R_{+}^{N},
$$

where $u_{(x, t)}$ is defined above. After some mathematical treatment we obtain that inequality (19) is true if

$$
\begin{aligned}
& \left(\tau+t_{*}\right)^{-\frac{N+n}{N m(p-1)+p-N}} \gg T^{-\frac{p-1}{q(p+n)-(p-1)(m(n+1)+1)}}, \\
& \left(\tau+t_{*}\right)^{-\frac{1}{N m(p-1)+p-N}} \ll T^{-\frac{q-m(p-1)}{q(p+n)-(p-1)(m(n+1)+1)}} .
\end{aligned}
$$

It implies that

$$
\frac{(p-1) /(N+n)}{q(p+n)-(p-1)(m(n+1)+1)}>\frac{q-m(p-1)}{q(p+n)-(p-1)(m(n+1)+1)},
$$

at $q<q_{c}$. Thus we obtain $u\left(x, t_{*}\right) \geqslant u_{1}\left(x, t_{*}\right) \geqslant u_{-}(x, 0)$. According to the comparison principle, solution $u(x, t)$ of problem $(1)-(3)$ becomes unbounded in finite time. 
Theorem 5. If $\frac{(N+n)(m+1)-n}{(N+n) m+1}<p<1+\frac{1}{m}$ then solution of problem (1)-(3) has the following asymptotic behavior

$$
u(x, t) \simeq C(T+t)^{-\gamma}\left(a+b \xi^{\frac{p+n}{p-1}}\right)^{-\frac{p-1}{1-m(p-1)}},
$$

when $\xi \rightarrow+\infty$ and $C=(\sigma((N+n)(m(p-1)-1)+p+n))^{1 /[1-m(p-1)]}$.

The critical case $(1-m(p-1)=0)$. The case $1-m(p-1)=0$ is called critical because the behavior of the solution is changed. In this case equation (1) has the following self-similar solution

$$
u_{+}(t, x)=(T+t)^{-\gamma_{c}} z(\xi)
$$

where $z(\xi)=e^{-d \xi^{\frac{p+n}{p-1}}}, \xi=|\zeta|, \zeta_{i}=\left(1+x_{i}\right)(T+t)^{-\sigma_{c}}, i=1,2, \ldots, N, \gamma_{c}=\frac{p-1}{(q-1)(p+n)}$, $\sigma_{c}=\frac{1}{p+n}, d=\frac{p-1}{m(p+n)}\left(\frac{1}{p+n}\right)^{1 /(p-1)}$. Using the comparison principle, we can prove that $u_{+}(x, t)=(T+t)^{-\gamma_{c}} z(\xi)$ is upper bound solution of problem (1)-(3) with sufficiently small initial data.

Theorem 6. Solution of problem (1)-(3) has the following asymptotic behavior

$$
u(t, x) \simeq C_{1}(T+t)^{-\gamma_{c}} z(\xi)
$$

when $\xi \rightarrow+\infty$ and $C_{1}$ is any positive number.

This result shows that problem (1)-(3) has no unique solution when parameters take critical values.

Theorems 5 and 6 can be proved in the same manner as it was done in [12-14].

Numerical experiments It is known that the choice of suitable initial approximation conserving nonlinear properties is very important in numerical analysis. For this purpose, we perform computer experiments on the basis of the obtained qualitative properties of solutions for the case of global solvability. Equation (1) is approximated with the second order of accuracy with respect to $\mathrm{x}$ and with the first order of accuracy with respect to $t$. Iterative process is proposed to obtain the approximate solution. During the inner steps of iteration the node values are calculated by the Thomas algorithm. It is well known that iteration methods require a good initial approximation. It allows one to achieve fast convergence to the exact solution and retain the qualitative properties of nonlinear processes. In what follows we present numerical scheme for the one-dimensional equation and some results of numerical experiments.

For convenience, we rewrite problem (1)-(3) as follows

$$
\begin{gathered}
\rho(x) \frac{\partial u}{\partial t}=\frac{\partial}{\partial x}\left(P(u) \frac{\partial u}{\partial x}\right), \quad(x, t) \in(0, b] \times(0, T), \\
-\left.P(u) \frac{\partial u}{\partial x}\right|_{x=0}=u^{q}(0, t), \quad t>0, \\
u(x, 0)=u_{0}(x) \geqslant 0, \quad x \in[0, b] \\
\left\{\begin{array}{l}
u(t, 0)=\phi_{1}(t)>0 \\
u(t, b)=\phi_{2}(t)>0
\end{array}\right.
\end{gathered}
$$

where $P(u)=u^{l-1}\left|u^{l-1} \frac{\partial u}{\partial x}\right|^{p-2}$. 
We approximate problem (22)-(24) by the implicit difference scheme that has the approximation error $O\left(h^{2}+\tau\right)$ :

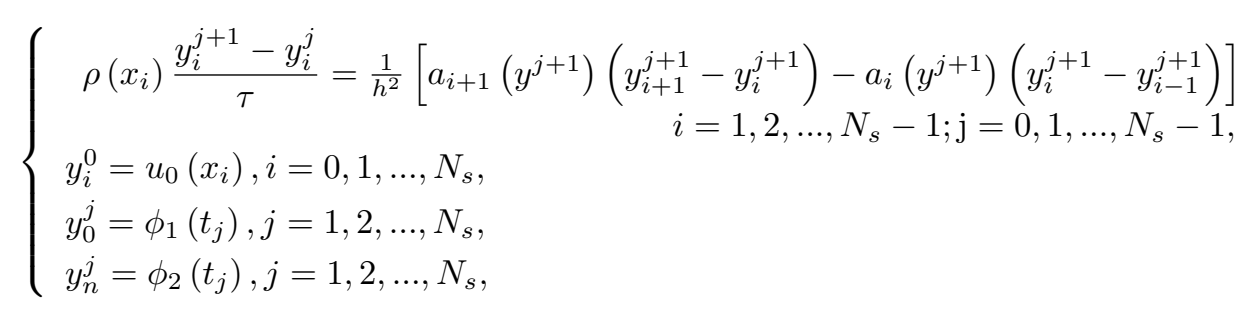

To ensure that difference scheme (26) is the second-order scheme the difference coefficient of thermal conductivity $a(y)$ should be calculated by one of the following formulae

$$
\begin{gathered}
a_{i}(y)=P\left(\frac{y_{i-1}+y_{i}}{2}\right), \\
a_{i}(y)=\frac{P\left(y_{i-1}\right)+P\left(y_{i}\right)}{2} .
\end{gathered}
$$

We set $\left.\frac{\partial u}{\partial x_{i}}\right|_{i=0} \approx \frac{y_{1}-y_{0}}{h}$ and $\left.\frac{\partial u}{\partial x_{i}}\right|_{i=n} \approx \frac{y_{n-1}-y_{n}}{-h}$ at the ends of interval $0 \leqslant x \leqslant b$. The system of algebraic equations (26) is nonlinear with respect to $y^{j+1}$. We use fixed-point iteration to solve the system of nonlinear equations:

$$
\rho\left(x_{i}\right) \frac{y_{i}^{j+1}-y_{i}^{j}}{\tau}=\frac{1}{h^{2}}\left[a_{i+1}\left(y^{j+1}\right)\left(\begin{array}{c}
s+1 \\
y_{i+1}^{j+1}-y_{i}^{j+1}
\end{array}\right)-a_{i}\left(y^{j+1}\right)\left(\begin{array}{c}
s+1 \\
y_{i}^{j+1}-y_{i-1}^{j+1}
\end{array}\right)\right]
$$

where $s=0,1,2, \ldots$ Difference scheme $(29)$ is linear with respect to $\stackrel{s+1}{y_{i}}$. The approximate solution from the previous time step is the initial guess for $\stackrel{s+1}{y}{ }_{i}: y^{j+1}=y^{j}$. The process is repeated until the condition

$$
\max _{0 \leqslant i \leqslant N_{s}}\left|\stackrel{s+1}{y}_{i}-\stackrel{s}{y}_{i}\right|<\varepsilon
$$

is satisfied. Let us introduce the following designations $y^{j}=y, y^{j+1}=\bar{y}$. Then, the difference equation can be rewritten as

$$
\stackrel{s}{A}_{i} \stackrel{s}{y}_{i-1}^{+1}-\stackrel{s}{C}_{i}{\stackrel{s}{y_{i}}}_{i}+\stackrel{s}{B}_{i} \stackrel{s}{y}_{i+1}^{+1}=-\stackrel{s}{F}_{i}, \quad i=1,2, \ldots, N_{s}-1,
$$

where $A_{i}=\frac{\tau}{h^{2}} a_{i}(y), B_{i}=\frac{\tau}{h^{2}} a_{i+1}(y), C_{i}=A_{i}+B_{i}+\rho\left(x_{i}\right), F_{i}=\rho\left(x_{i}\right) y$. Coefficients $a_{i}(y)$ are calculated according to $(29)$ :

$$
a_{i}(y)=\frac{1}{2}\left[\left(y_{i}\right)^{l-1}\left|\left(y_{i}\right)^{l-1} \frac{y_{i}-y_{i-1}}{h}\right|^{p-2}+\left(y_{i-1}\right)^{l-1}\left|\left(y_{i-1}\right)^{l-1} \frac{y_{i-1}-y_{i-2}}{h}\right|^{p-2}\right] .
$$

The Thomas method is used for the numerical solution of equations (30). Now we present the results of numerical experiments. Parameters of computational scheme: grid size $h=0.05$, number of nodes $N_{h \tau}=10000$ and convergence tolerance $\varepsilon=10^{-3}$. Process duration $t=4$ and the time step $\tau=0.04$. In all figures dashed lines correspond to the initial approximation at $t=0$ and $t=t_{N_{s}}=4$ and continuous lines correspond to numerical solutions.

The initial approximation for iterative process is described by expression (20). 
Fig. 1 shows the numerical solution of problem (1)-(3) at $q>q_{c}$. In this case the speed of propagation of the disturbance is infinite. Due to the unbounded filtration coefficient, the speed of propagation of the disturbance is much larger than in the case of slow diffusion $(p>1+1 / m)$ which is marked by the finite speed of propagation of the disturbance. The process of filtration exists in the whole region and vanishes at infinity. Fig. 2 shows the numerical solution near the critical point $1-m(p-1) \rightarrow 0$.

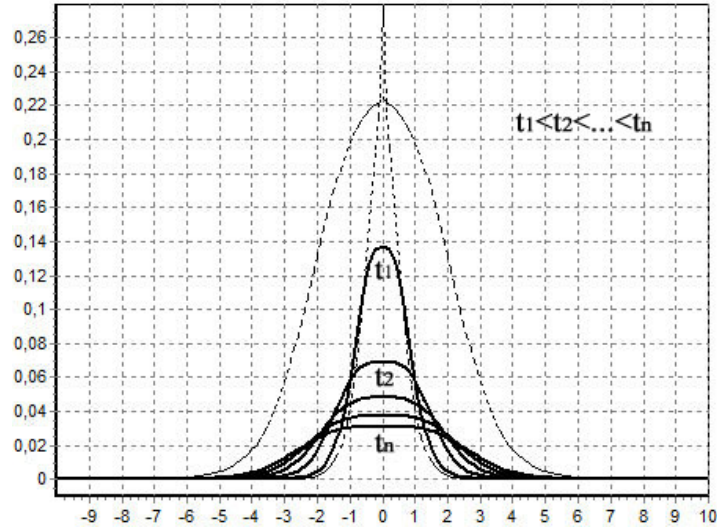

a)

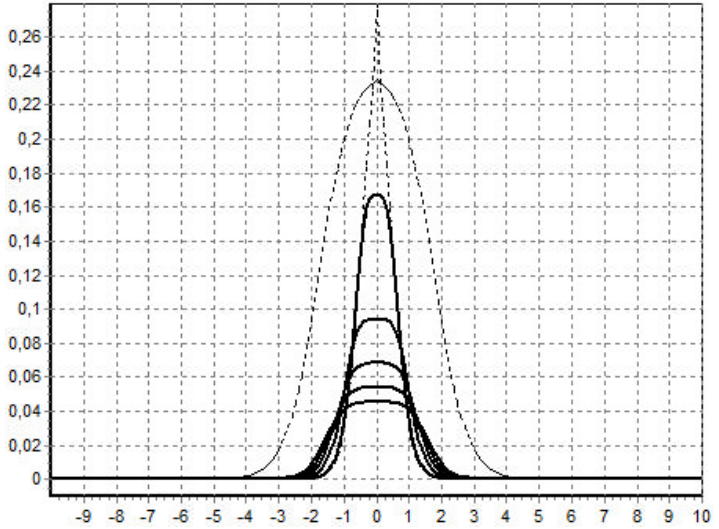

b)

Fig. 1. $m=1.5, p=1.55, q=2.85$, a) $n=0.5$, b) $n=1$

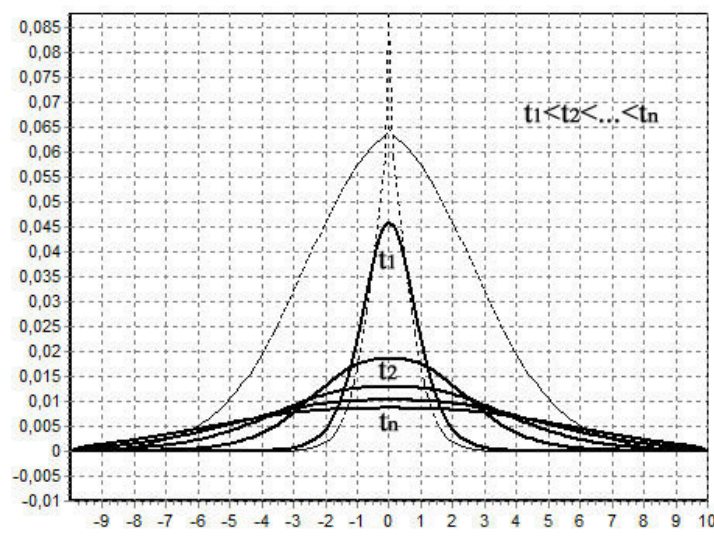

a)

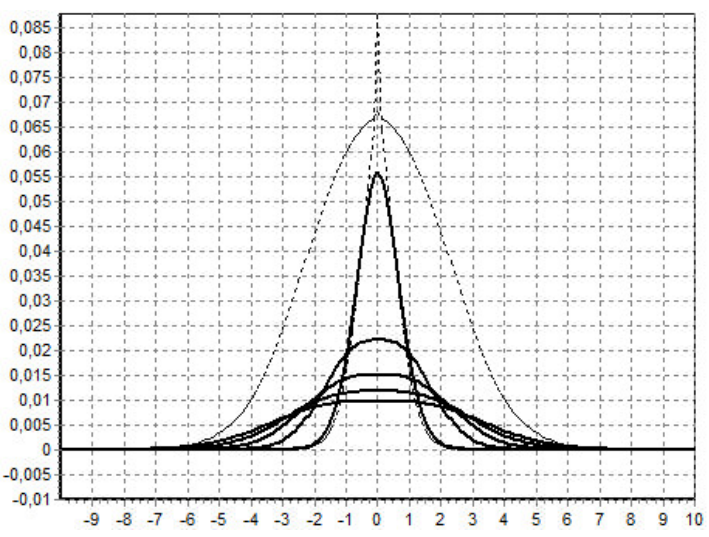

b)

Fig. 2. $m=1.5, p=1.61, q=3$, a) $n=-0.1$, b) $n=0.25$

The initial approximation for iterative process is described by expression (21).

All figures show that filtration process dependents on the density of medium. Numerical experiments show fast convergence of iterations to the exact solution. It is due to the choice of a suitable initial approximation. The number of iterations is not more than 6 for various values of numerical parameters. 


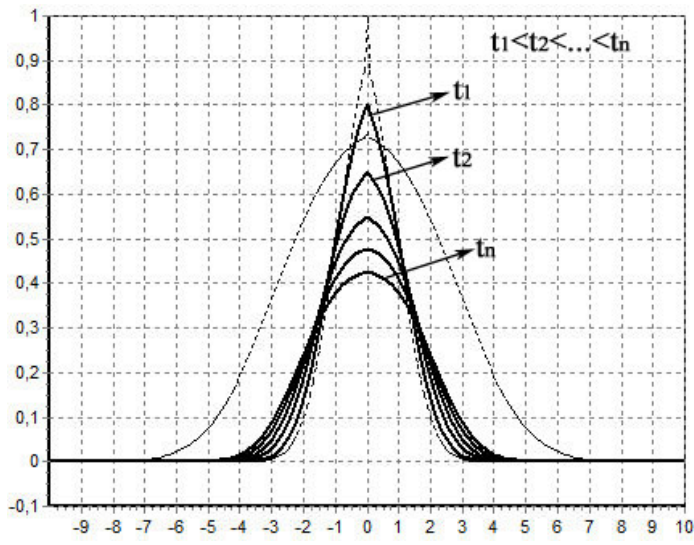

a)

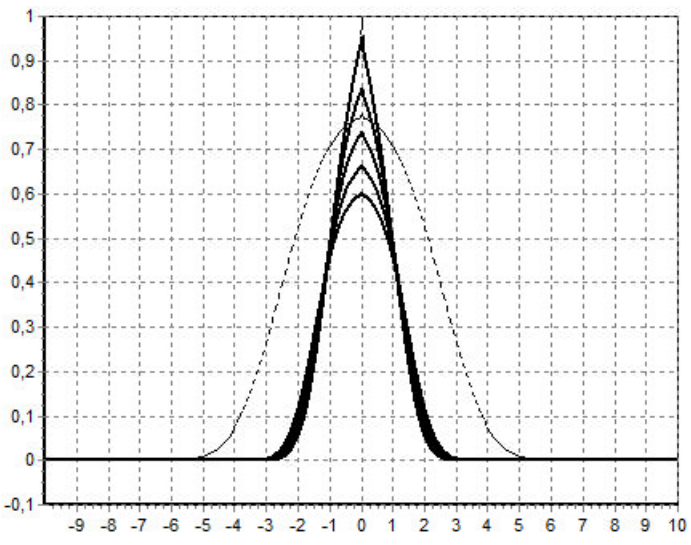

b)

Fig. 3. $m=1.25, p=1.8, q=2.85$, a) $n=0.5$, b) $n=1$

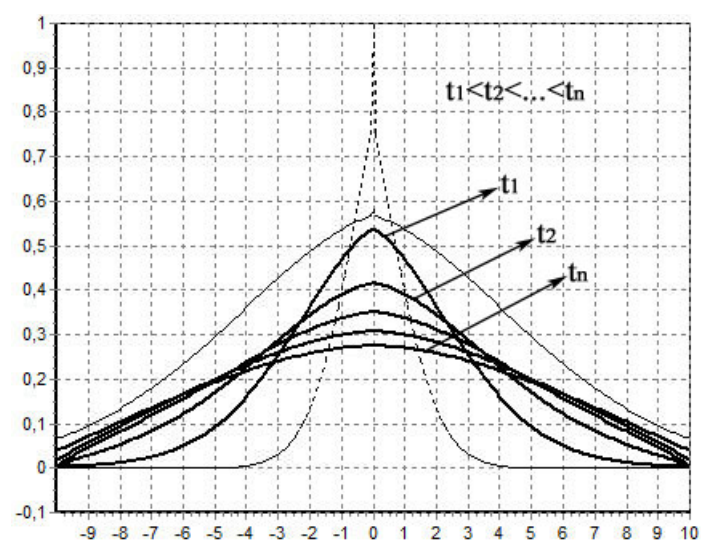

a)

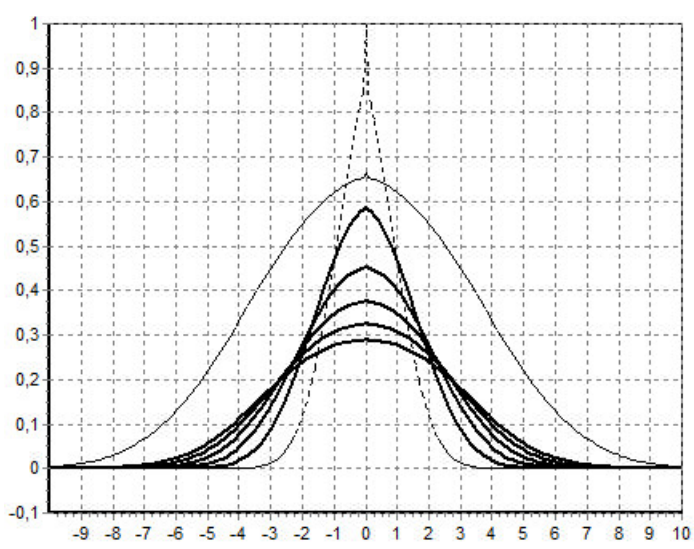

b)

Fig. 4. $m=1.25, p=1.8, q=2.85$, a) $n=-0.25$, b) $n=0.25$

\section{References}

[1] M.M.Aripov, Standard Equation's Methods for Solutions to Nonlinear problems, (Monograph), FAN, Tashkent, 1988 (in Russian).

[2] A.S.Kalashnikov, Some problems of the qualitative theory of nonlinear degenerate secondorder parabolic equations, Russian Math. Surveys, 42(1987), 169-222.

[3] V.A.Galaktionov, J.L.Vazquez, The problem of blow-up in nonlinear parabolic equations, Discrete and continuous dynamical systems, 8(2002), no. 2, 399-433.

[4] C.P.Wang, S.N.Zheng, Critical Fujita exponents of degenerate and singular parabolic equations, Proc. Roy. Soc. Edinburgh, Sect. A, 136(2006), no. 2, 415-430.

[5] W.Zejia, Y.Jingxue, W.Chunpeng, Critical exponents of the non-Newtonian polytropic filtration equation with nonlinear boundary condition, Appl. Math. Lett., 20(2007), 142-147.

[6] Z.Li, Ch.Mu, Critical exponents for a fast diffusive polytrophic filtration equation with nonlinear boundary flux, J. Math. Anal. Appl., 34(2008), 55-64. 
[7] C.Jin, J.Yin, Critical exponents and non-extinction for a fast diffusive polytrophic filtration equation with nonlinear boundary sources, Nonlinear Anal., 67(2007), 2217-2223.

[8] P.Zheng, Ch.Mu, D.Liu, X.Yao, Sh.Zhou, Blow-up analysis for a quasilinear degenerate parabolic equation with strongly nonlinear source, Abstract and Appl. Anal., 2012(2012), Article ID 109546.

[9] W.Du, Z.Li., Critical exponents for heat conduction equation with a nonlinear Boundary condition, Int. Jour. of Math. Anal., 7(2013), no. 11, 517-524.

[10] Li Z., Mu Ch. and Du W., Critical Fujita exponent for a fast diffusive equation with variable coefficients, Bull. Korean Math. Soc., 50, no. 1 (2013), 105-116.

[11] V.A.Galaktionov, H.A.Levine, On critical Fujita exponents for heat equations with nonlinear flux boundary condition on the boundary, Israel J. Math., 94(1996), 125-146.

[12] M.M.Aripov, Z.R.Rakhmonov, On the asymptotic behavior of the self-similar solutions of a nonlinear problem of polytropic filtration with nonlinear boundary conditions, Vychislitel'nye tekhnologii, 18(2013), no. 4, 50-55 (in Russian).

[13] M.Aripov, Z.Rakhmonov, Numerical simulation of a nonlinear problem of a fast diffusive filtration with a variable density and nonlocal boundary conditions, Mathematical Models and Simulation in Science and Engineering, Ser. 23, (2014), 72-77.

[14] Z.R.Rakhmonov, On the behavior of solutions of the problem of nonlinear filtration with a variable density and nonlocal boundary condition, Uzbek. Matem. Zurnal, (2015), no. 1, 75-85 (in Russian).

[15] Z.R.Rakhmonov, On one nonlinear problem of non-Newtonian Filtration in an inhomogeneous medium with nonlocal boundary conditions, KazNU Bulletin, (2014), no. 3, 45-56 (in Russian).

\title{
К свойствам решений одной многомерной задачи нелиней- ной фильтрации с переменной плотностью и нелокальным граничным условием в случае быстрой диффузии
}

Зафар Р. Рахмонов

Национальный университет Узбекистана ул. Университет, 4, 100174

Узбекистан

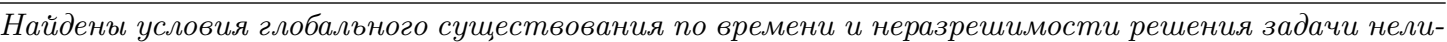
нейной фильтрации в неоднородной среде на основе метода эталонных уравнений, автомодельного анализа и метода сравнения решений. Изучено влияние неоднородности среды на эволючию процесса. Получена критическая экспонента типа Фужита и критическая экспонента глобального существования по времени решения. В случае глобальной разрешимости получен главный член асимптотики решений на бесконечности.
\end{abstract}

Ключевые слова: фильтрация, асимптотика, критическая экспонента, режим с обострением, неоднородная плотность. 